\title{
Decreased volume of the cerebral ventricles on CT images in Gilles de la Tourette's syndrome
}

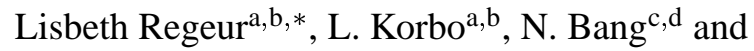 \\ B. Pakkenberg ${ }^{\mathrm{a}, \mathrm{b}}$ \\ ${ }^{a}$ Neurological Research Laboratory, Bartholin \\ Institute, Kommunehospitalet, Copenhagen, Denmark \\ ${ }^{\mathrm{b}}$ Stereological Research Laboratory, Aarhus \\ University, Århus, Denmark \\ ${ }^{\mathrm{c}}$ Department of Radiology, Roskilde County Hospital, \\ Roskilde, Denmark \\ ${ }^{\mathrm{d}}$ Department of Radiology, Gentofte County Hospital, \\ Hellerup, Denmark
}

\begin{abstract}
The aim of the present study was to estimate the volume of the ventricular system comprising lateral plus third ventricles in patients with Gilles de la Tourette's syndrome on computed tomographic (CT) scannings using unbiased stereological principles and to compare that volume with a control group. We found a significantly reduced ventricular volume in 24 patients with Gilles de la Tourette's syndrome (GTS) compared with 28 controls.
\end{abstract}

Keywords: CT-scanning, stereology, tics, Tourette's syndrome, ventricular volume

\section{Introduction}

Gilles de la Tourette's syndrome (GTS) is a neurobehavioural disorder of childhood onset, characterized by motor and phonic tics that wax and wane in severity. Neuropathologic, neuroanatomic, and neuroradiologic data have pointed to a variety of brain regions as target areas including the basal ganglia [15] and related frontal lobe regions [1], the limbic system [2], periaqueductal grey and midbrain tegmentum [4], and to changes of the ventricular volume [14]. Neuroradiographic studies with $\mathrm{CT}$ and magnetic resonance imaging (MRI) have identified isolated defects such as mild ventricular dilation, prominent sylvian fissures,

${ }^{*}$ Corresponding author: Lisbeth Regeur, Neurological Research Laboratory, Bartholin Institute, Københavns Kommunehospital, 1399 Copenhagen K, Denmark. Tel.: +45 333838 42; Fax: +45 33383843 . and cysts [16]. Peterson et al. [12], using reconstructed nonoverlapping, proton-density-weighted MR images in 14 GTS patients and 14 controls, reported volume reduction for the left but not the right-sided lenticular nucleus. Post hoc analyses revealed smaller mean volumes of the caudate, lenticular, and globus pallidus nuclei compared with controls on both the right and left side. The volumetric basal ganglia asymmetry Peterson et al. [12] found in controls, could not be displayed in the GTS patients.

Singer et al. [16] performed a volumetric MRI study of basal ganglia structures and lateral ventricles in 37 children with GTS and 18 controls. They found significant differences for measures of symmetry in the putamen and the lenticular region, since virtually all controls had a left-sided predominance of the putamen, whereas in 13 of 37 GTS subjects, a right predominance exceeded that of any control. No statistically significant differences were found in the size of the right or left caudate, putamen, globus pallidus, or ventricles in these populations. Hyde et al. [9] performed morphometric analyses of MRI's of 10 monozygotic twin pairs (mean age 16.3 years) discordant for severity of GTS but concordant for the presence of tic disorders. Right caudate volume was slightly but significantly reduced in the relatively more severely affected twins as a group compared with the less affected twins. Most of this difference was attributable to volume reduction in the anterior right caudate, which was smaller in the more severely affected twin in nine of 10 twin sets. The mean volume of the left lateral ventricle was $16 \%$ smaller in the more severely affected twins than in the less severely affected. The (expected) asymmetry of the lateral ventricles was not present in the more severely affected twins, who had a trend towards a larger right lateral ventricle $[6,8,12]$. Within a pair, the difference in the degree of loss of the normal ventricular asymmetry correlated with the difference in the severity of the tic disorder. There were no other basal ganglia, ventricular volumetric or asymmetry abnormalities. 
The aim of the present study was to obtain estimates of the volume of the ventricular system using a stereological method based on unbiased principles for volume measurements from CT images and to compare that volume with a control group on CT-scans.

\section{Material}

Twenty-four patients with GTS, 19 males and 5 females, with an average age of 16.8 years (7-37 years), were scanned from 1977-1983 and compared with 28 controls, 23 males and 5 females with an average age of 18.0 years (9-29 years), scanned from 1987 to 1995 . Originally 53 patients with GTS were scanned from 1977-1983, but due to changes of the daily routine, only the first 32 of these 53 scans were copied and saved. A further 8 GTS cases were excluded because the images were severely degraded by motion artifact. Of the remaining 24 CT-scans, 21 were scanned at Hvidovre University Hospital, 2 at Esbjerg County Hospital, and one at Rigshospitalet, and all were measured using a stereological method and compared with CT-scans from 28 controls from two county hospitals in Denmark, the County Hospital in Roskilde and the County Hospital in Gentofte.

Average disease duration in the 24 GTS patients was 9.8 years $(1-33)$. For all clinical data pertaining to the GTS patients, see Table 1. The control cases, 23 males and 5 females, with a mean age of 18.0 years (9-29) were scanned in an outpatient clinic because of complaints about temporary headache (17), vertigo (5), vision disturbances (3), head trauma (2), and one epileptic episode (1). In all controls and all GTS cases the diagnosis of a normal CT-scan was given by an experienced neuroradiologist.

\section{Method}

\subsection{CT-scanning}

Twenty-one GTS patients were CT-scanned on a $160 \times 160$ matrix 1010 EMI scanner with water bag, operated at $120 \mathrm{kV}, 33 \mathrm{~mA}$ with a scantime of $4.5 \mathrm{~min}$. The minification factor was 2.27 at the two general community hospitals and 4.16 at Hvidovre University Hospital. The GTS patient from Rigshospitalet was scanned on a $160 \times 160$ matrix EMI head scanner Mark 1 , operated at $120 \mathrm{kVp}$ and $33 \mathrm{~mA}$ with a minification factor of 3.3 and a scantime of $4 \mathrm{~min}$., and the two patients from Esbjerg on a Siemens Somatom 2 with a minification factor of 3.7 , scantime 7 seconds. The control cases were scanned on two $256 \times 256$ matrix scanners, a PACE and a General Electric 9800. Both scanners operated at $140 \mathrm{kV}$ and $120 \mathrm{~mA}$ with a scantime of 3 seconds. The scan plan thickness was $10 \mathrm{~mm}$ in all cases and so was the distance between each scan plan.

\subsection{Stereology}

The estimation of the volume of the ventricular system was performed on an average of 4.0 scan plans (2$5)$. The polaroid pictures were transferred to a videoscreen via a video camera. A computer generated counting grid with an area per point, $a(p)=18 \mathrm{~mm}^{2}$, was superimposed randomly on the scan picture at the video screen using the CAST-grid $\circledR$ system (Olympus, Denmark) (Fig. 1). All points hitting the third plus lateral ventricles were counted.

An estimate of the total ventricular area was obtained by counting all points hitting the ventricular system, $\sum P$, and multiplying that sum with the corresponding area, $a(p)$ per point. The scans were made axially, parallel to the orbitomeatal axis and were randomized, so that the first slice hitting the ventricles fell randomly, followed by systematic sections with a known fixed interval equal to the scan plan thickness, $t=10 \mathrm{~mm}$. For an unbiased volume estimation, the grid must be thrown with a random position, and for estimation of the coefficient of error, $\mathrm{CE}=\mathrm{SEM} /$ mean, the grid must be rotated isotropically as well [5]. According to the Cavalieri method, an estimate of the ventricular volume, $V$ (ventricular system), can be obtained by multiplying the estimate of the area by the scan plane thickness [10]:

$$
V(\text { ventricular system })=t \times a(p) \times \sum P .
$$

The volume of the ventricular system is usually underestimated on a scan plane of several $\mathrm{mm}$ because of underprojection. The bias of underestimation is limited by using a correction-factor, the maximal number of points counted on one section, $P(\max )$ [3]. Both $a(p)$ and $t$ were constants within each brain. The final estimate of each ventricular system is thus equal to:

$$
\begin{aligned}
& V(\text { ventricular system })= \\
& t \times a(p) \times\left[\sum P+P(\text { max })\right] .
\end{aligned}
$$

The average number of counted points in controls was 35 (14-79), and in GTS patients 40 (14-98). The $\mathrm{CE}$ on the estimate was 0.15 for the controls and 0.19 for the GTS patients. 


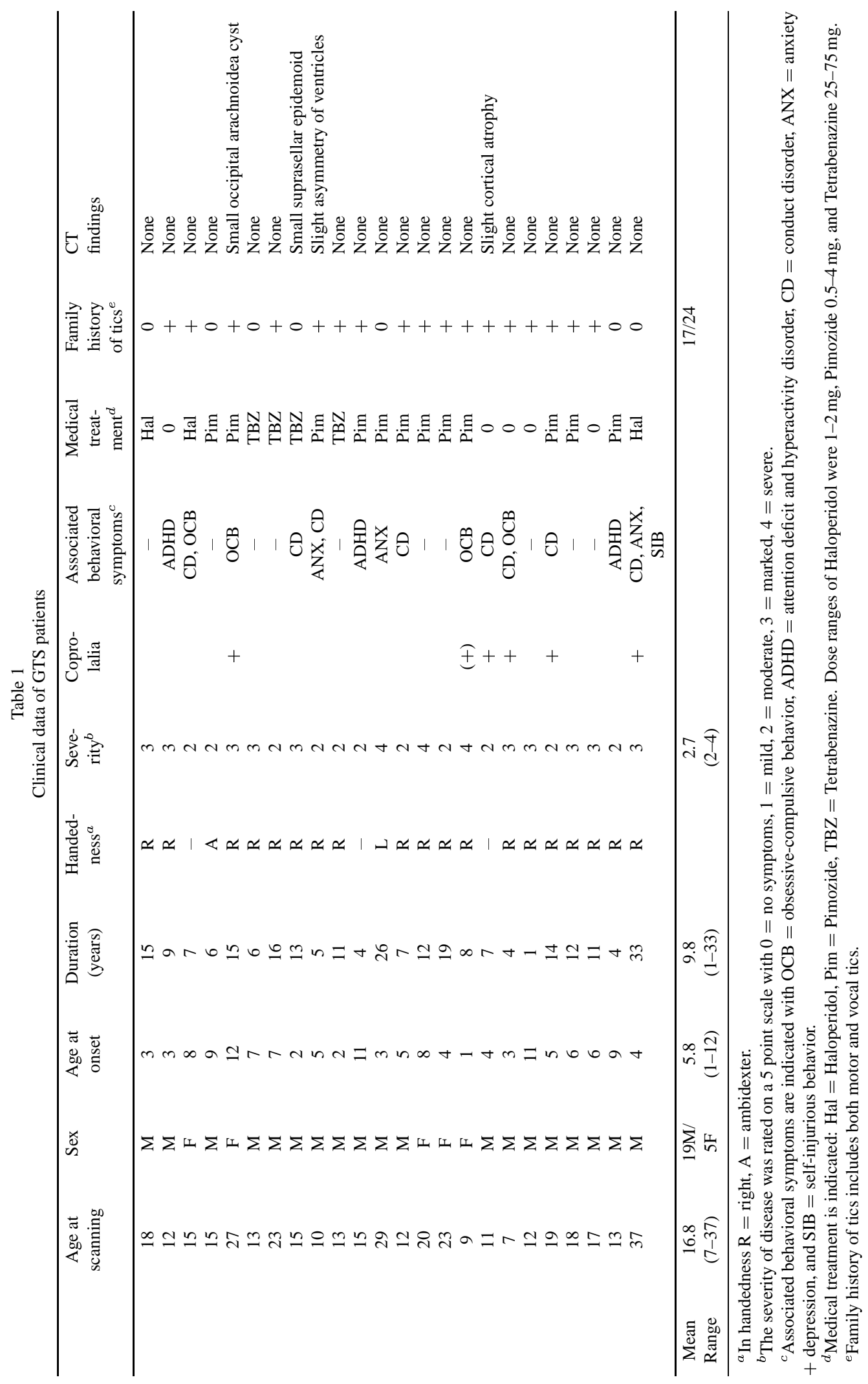




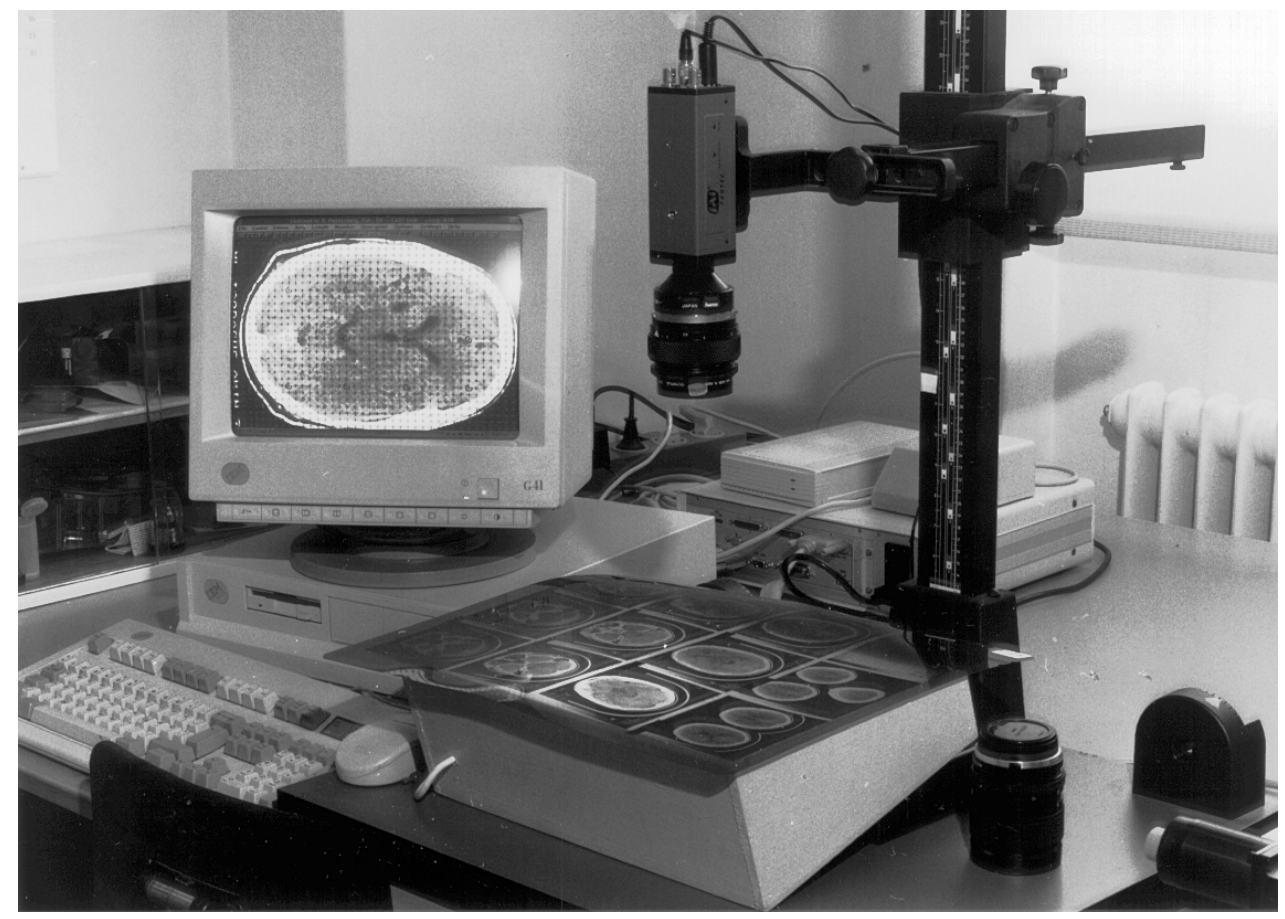

Fig. 1. Shows a video camera that transfers the polaroid pictures to a video-screen. A counting grid is superimposed on the screen with a random position, and all points hitting the third plus the lateral ventricles are counted.

Since the subjects in the study ranged in age from 7 to 37 years in patients and from 9 to 29 years in controls, we measured the cranial circumference at the level of $2 \mathrm{~cm}$ above the Frankfurt plan. The circumference was estimated from a set of parallel testlines placed randomly over the selected scan plan:

$$
\text { cranial circumference }=\pi / 2 \times h / 2 \times \sum I,
$$

where $h$ is the distance between the parallel lines (in this case $5 \mathrm{~mm}$, before correction for the linear magnification), and $\sum I$ is the sum of intersections between the outer cranial circumference and the testlines.

\subsection{Statistics}

The distributions of ventricular volume turned out to be log-normal, and therefore the geometric mean value is reported. The coefficient of variation $(\mathrm{CV})$ was thus $=\exp (\mathrm{SD}[\log$ vol $])-1$ and the $\mathrm{CE}=$ $\exp (\mathrm{SEM}[\log$ vol $])-1$. Student's $t$-test was used to evaluate differences between the groups. With a CV varying from 0.41 to 1.57 , the precision of the estimate was more than sufficient. Intra-observer variability is not estimated but is expected to be close to the CE.

\section{Results}

The volumes of the ventricles can be seen in Fig. 2 . The geometric mean ventricular volume was 14.0 $(\mathrm{CV}=0.90) \mathrm{cm}^{3}$ in the control males and statistically significantly smaller, $12.2(\mathrm{CV}=0.75) \mathrm{cm}^{3}$, in the GTS group, $p=0.04$. The mean volume of the ventricular system in the GTS females (9.71 $\left.(\mathrm{CV}=0.41) \mathrm{cm}^{3}\right)$ was rather close to that of the control females $\left(10.7(\mathrm{CV}=1.57) \mathrm{cm}^{3}\right)$ (see Fig. 2) but the number of females in the control group $(n=5)$, and the GTS female group $(n=5)$ was too small to make a meaningful comparison. The females were therefore not included in the analyses concerning cranial circumference and age effect.

The youngest control boy was 9 years old and the oldest male 29 years. The two largest volumes were found in two control males, age 19 and 21. The increase in cranial circumference as a function of age was statistically significant for the control males, $r=0.60$, $2 p=0.0025$, while this was not the case for the GTS patients, $r=0.18,2 p=0.47$, see Fig. 4 . The ventricular volume was positively correlated to cranial circumference only in the control males, $r=0.43$, $2 p=0.043$ with $r=0.31,2 p=0.21$ for the GTS patients. 


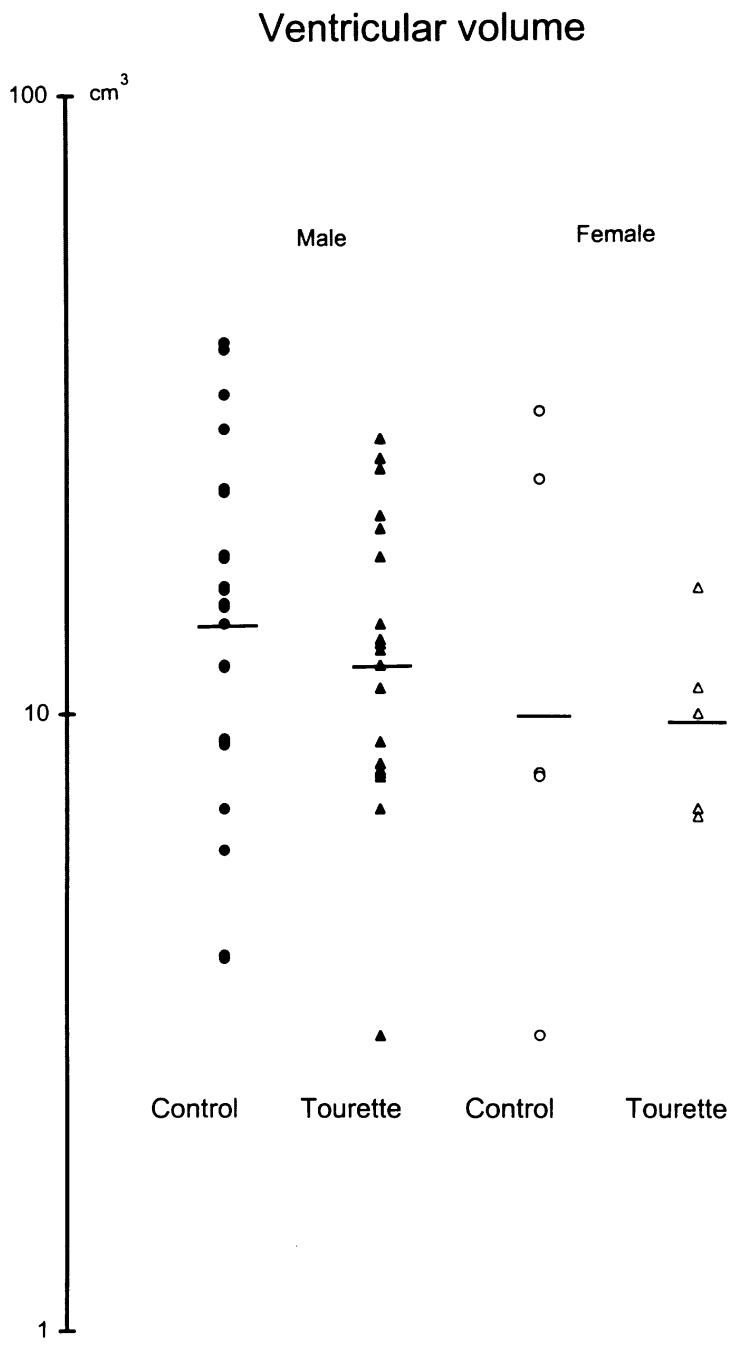

Fig. 2. The ventricular volume of the GTS patients divided into males $(\boldsymbol{\Delta})$ and females $(\triangle)$, and a control group of males $(\bullet)$ and females $(\mathrm{o})$ is shown on a logarithmic scale.

\section{Discussion}

The reduced ventricular volume in the 24 cases with Tourette's syndrome was unexpected, since it is unusual to find decreases in the ventricular volume in neurological/psychiatric disorders. According to Robertson [14], only 18 out of 172 documented CTscans performed on patients with GTS have been abnormal, and only rarely the abnormalities appear to be of direct aetiological significance. None of the former CT-scan studies, independent of quantitative, semiquantitative or qualitative method, have found reduced ventricular size in GTS patients. Harcherik et al. [8], for example, used CT-scanning in four neuropsychiatric disorders of childhood, among these GTS. Eigh- teen male and one female, mean age 19.6 years, with GTS were compared with 20 controls, 12 male, eight female, mean age 11.1 years. Ventricular volume and brain density were determined by quantitative, computer-based methods by researchers blind to the patients' diagnoses. Brain density, the number generated by computer representing the mean denseness for the region of interest, was computed for three slices from each hemisphere. Level A was the highest slice in which lateral ventricles were seen, and levels B and $\mathrm{C}$ were the next two higher contiguous slices. No significant differences among GTS and controls were found in total ventricular volume, right-left ventricular volume ratio, ventricular asymmetrics, ventricle-brain ratios, or brain density.

The male GTS patients had smaller lateral plus third ventricular size, the cranial circumference was not related to age, and the ventricular volume was not related to cranial circumference. Because the smaller lateral plus third ventricular size is not correlated to head size, there is no evidence supporting that the smaller ventricular size in the GTS patients compared to the controls was caused by smaller heads. Not unexpectedly, the size of the lateral plus third ventricle was positively correlated to cranial circumference in the controls, and the cranial circumference is positively correlated to age.

In our previous study of 65 GTS patients [13], in which the present 24 patients are included, the first 53 had a CT-scanning as part of their clinical evaluation. Forty-seven of these were judged to be normal according to standard neuroradiological criteria. Abnormalities included a small arachnoid cyst in the occipital region, slight cortical atrophy, small suprasellar epidemoid (Table 1) and two cases showing asymmetry of the ventricles. The asymmetry is only based on a qualitative judgement. The statistically significant reduction in the ventricular system found in this study was only revealed by actually estimating the volumes in a stereological design.

Some features of this study are not optimal:

1. Since GTS is characterized by multiple motor tics, it can be difficult/impossible to obtain a sufficiently high quality of the CT-images. This problem was overcome by excluding all CTscans where the quality of the scan was considered to be a serious limitation. This could result in a selection bias, since GTS patients with the most severe motor tics of the head were excluded. However, although severity of the tic disorder has been related to ventricular parame- 


\section{Venticular volumen in}

male controls

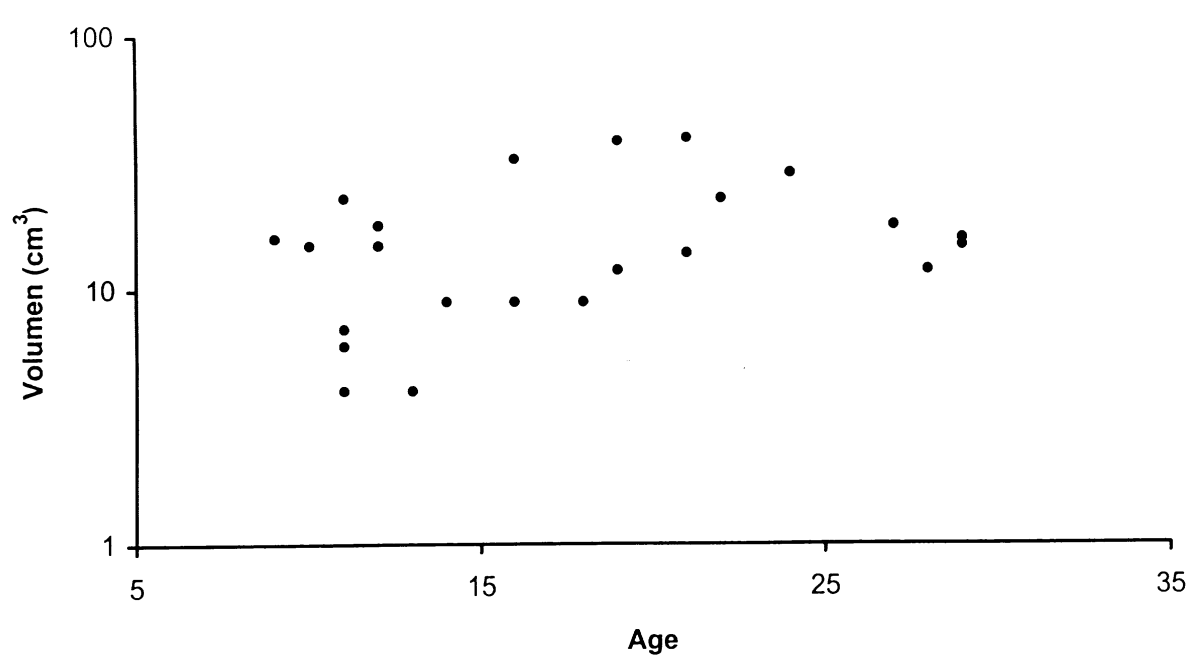

Fig. 3. Shows that there is no correlation between ventricular volume and age. The two largest volumes are found in control males, aged 19 and 21 .

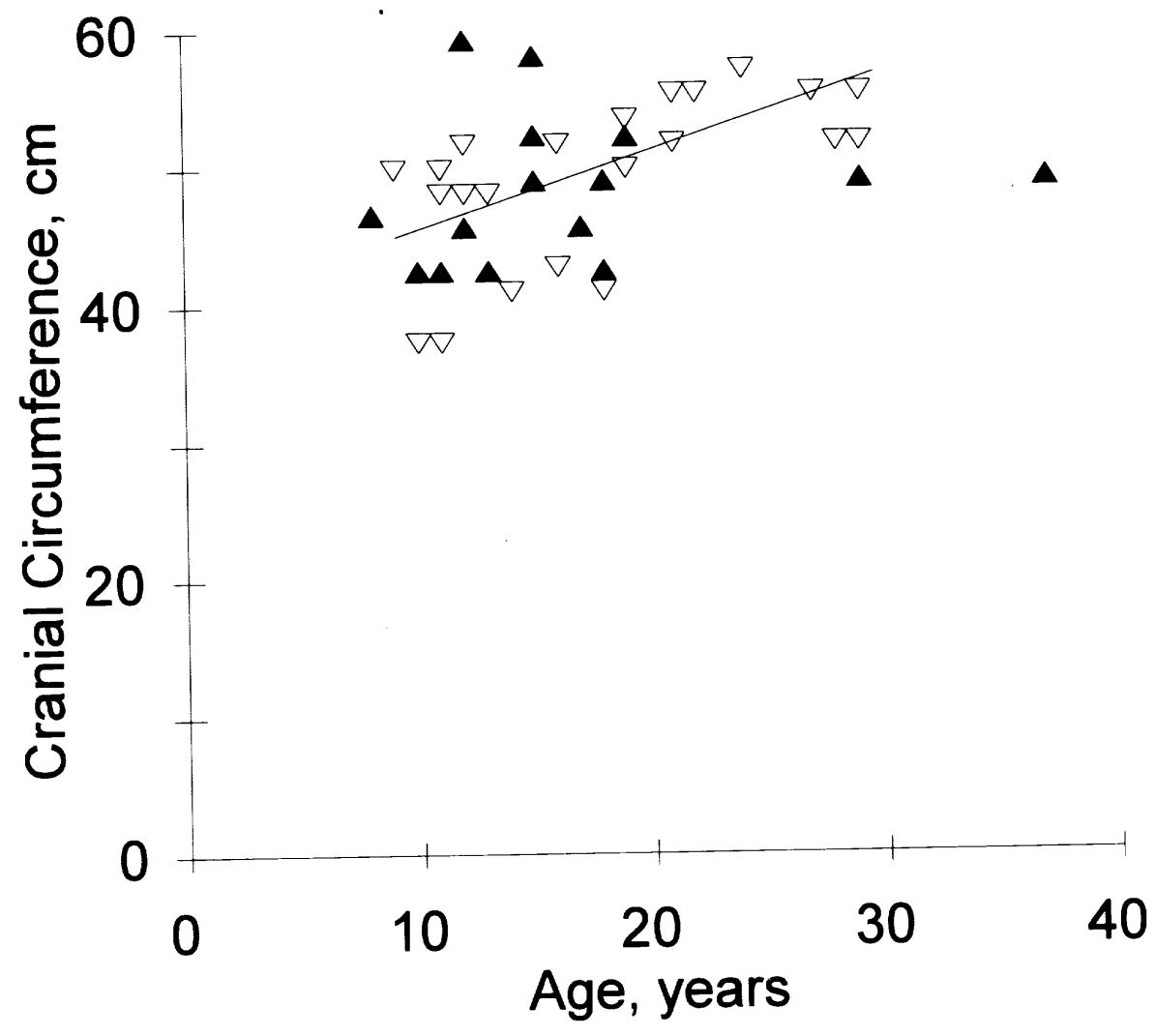

Fig. 4. Shows the cranial circumference in $\mathrm{cm}$ as a function of age. $(\boldsymbol{\Lambda})$ GTS males, $(\nabla)$ control males. 


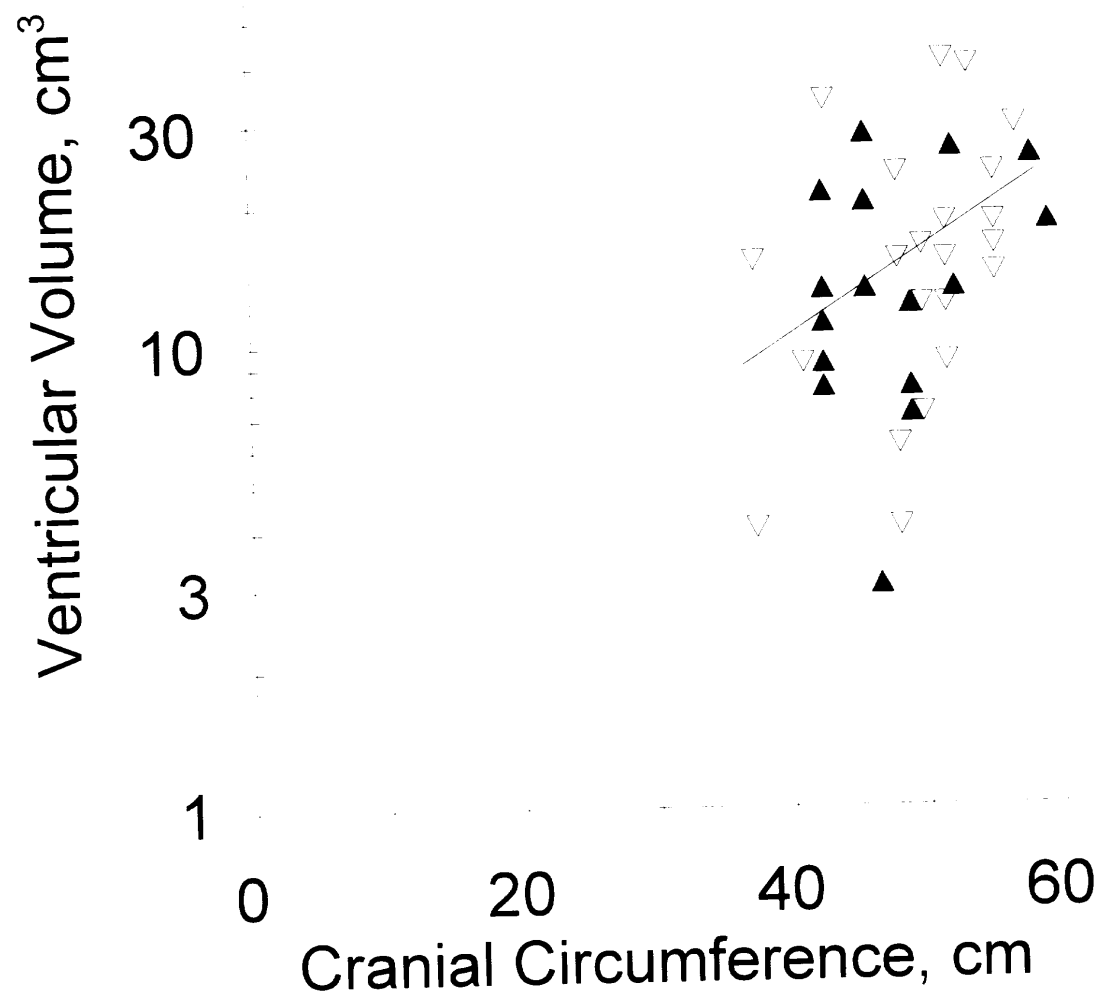

Fig. 5. The relation between the volume of the lateral plus third ventricles $\left(\mathrm{cm}^{3}\right)$ and cranial circumference $(\mathrm{cm})$. The ventricular volume is positively correlated to cranial circumference only in the control males. $(\boldsymbol{\Delta})$ GTS males, $(\nabla)$ control males.

ters, this has been in relation with possible lack of asymmetry more than with the volumetric size in the GTS patients [9].

2. The control cases were scanned mostly because of short, temporary headache, vertigo, or visual disturbances. It can be discussed whether they represented an appropriate control group. However, since we did not have access to strictly normal CT-scans from approximately the same time period in this retrospective study, only those with mild and temporary symptoms were chosen as controls. Furthermore, all CT-scannings from the control cases were diagnosed as normal by an experienced neuroradiologist.

3 . Because of several reports of changes in ventricular asymmetry (the left larger than right imbalance disappears) $[6,8,9,12]$, it was part of the original protocol to estimate the lateral ventricles and the third ventricle separately. However, the ventricular system is a rather small part of the brain and would in certain cases only show-up on two scan-pictures. Since we know that the biological variance of the volume of the ventricular system is high among human individuals [11], which is also reflected in the high coefficient of variation in the present study, we decided only to estimate the total volume of the lateral plus the third ventricles.

4. Different scanners were used for the GTS patients and the controls. The ventricles, however, are not difficult to define on a CT-scanning, and the use of a variety of scanners gives estimates of the size of the ventricular system, which are not based on a single CT-scanner and therefore provides, on average, a more general and useful mean value.

Because of the wide variation of the ventricular volume in the GTS and in the control group, estimation of the ventricular volume in an individual situation is not of clinical significance. It is, however, interesting that as a group, patients with GTS seem to have a smaller 
volume of the lateral plus third ventricles than controls. Postmortem, reduced dynorphin-like staining in the external globus pallidus and substantia nigra has been reported in one case of GTS [7]. Furthermore, it can be seen from Fig. 2 that the normal spread around the mean is smaller in the GTS cases than in controls, which would indicate that the size of the ventricular system in GTS patients is more homogeneous than in controls.

The advantages of the present study include:

1. The use of stereological methods ensure that the estimates are obtained in a design based on random systematic point counting providing estimates of volume based on unbiased principles.

2. Reporting estimates of the real volume of the ventricular system excludes the many problems related to data expressing the size of the ventricular volume based on 2-dimensional measurements of ventricular areas or linear measurements. As an example, in a series of 10 patients who were studied by magnetic resonance imaging (MRI), the effect of 1-mm variations in slice center level on measured $2 \mathrm{D}$ ventricle-brain ratio (VBR) on a single 'best' section was determined; even variations as small as $1 \mathrm{~mm}$ could result in a $10 \%$ change in VBR [17]. Furthermore, the angle at which the patient is scanned has no influence on the estimate of the volume, since the volume estimate is independent of the angle of the scan plan. Finally, we applied a correctionfactor to limit the bias of underestimation of the ventricular volumes due to the thickness of the scan plane.

3. The decision whether a point on the pointcounting grid is inside or outside the ventricular system is based on manual counts, which means that the design is rather insensitive to thresholding levels of grey matter to define brain parenchyma versus CSF space and to CTscanning artifacts which is a potential problem in other studies.

Evidently, great care should be taken when interpreting results in findings as unspecific as a change in the size of the ventricular system. A first step of interest could be to have the ventricular system measured by others using the same or equally valid methods to judge the reproducibility of the finding, preferably on more modern imaging equipment.

\section{Acknowledgements}

Dr. Agnete Karle, Department of Radiology, Bispebjerg Hospital is sincerely thanked for performing most of the CT-scans and Hans Jørgen G. Gundersen, Stereological Research Laboratory, Aarhus University for excellent reviewing and statistical aid. Furthermore we thank the Hartmann Brothers Foundation for financial support.

\section{References}

[1] M.L. Berthier, A. Bayes and E.S. Tolosa, Magnetic Resonance Imaging in Patients with Concurrent Tourette's Disorder and Asperger's Syndrome, Journal of the American Academy of Child and Adolescent Psychiatry 32 (1993), 633639.

[2] T.N. Chase, V. Geoffrey, M. Gillespie and G.H. Burrows, Structural and Functional Studies of Gilles de la Tourette Syndrome, Revue Neurologique (Paris) 142 (1986), 851-855.

[3] R. Coleman, The sizes of spheres from profiles in a thin slice. II. Transparent spheres, Biometrical Journal 25 (1983), 745-755.

[4] O. Devinsky, Neuroanatomy of Gilles de la Tourette's Syndrome, Archives of Neurology 40 (1983), 508-514.

[5] H.J.G. Gundersen and E.V. Jensen, The efficiency of systematic sampling in stereology and its prediction, Journal of Microscopy 147 (1987), 229-263.

[6] C. Gyldensted, Measurements of the normal ventricular system and hemispheric sulci of 100 adults with computed tomography, Neuroradiology 14 (1977), 183-192.

[7] S.N. Haber, N.W. Kowall, J.P. Vonsattel, E.D. Bird and E.P. Richardson, Gilles de la Tourette's syndrome: a postmortem neuropathological and immunohistochemical study, Journal of Neurological Science 75 (1986), 225-241.

[8] D.F. Harcherik, D.J. Cohen, S. Ort, R. Paul, B.A. Shaywitz, F.R. Volkmar, S.L.G. Rothman and J.F. Leckman, Computed Tomographic Brain Scanning in Four Neuropsychiatric Disorders of Childhood, American Journal of Psychiatry 142 (1985), 731-734.

[9] T.M. Hyde, M.E. Stacey, R. Coppola, S.F. Handel, K.C. Rickler and D.R. Weinberger, Cerebral Morphometric Abnormalities in Tourette's Syndrome: A Quantitative MRI Study of Monozygotic Twins, Neurology 45 (1995), 1176-1182.

[10] B. Pakkenberg, J. Boesen, M. Albeck and F. Gjerris, Unbiased and Efficient Estimation of Total Ventricular Volume of the Brain Obtained from CT-Scans by a Stereological Method, Neuroradiology 31 (1989), 413-417.

[11] B. Pakkenberg and H.J.G. Gundersen, Neocortical neuron number in humans: effect of sex and age, Journal of Comparative Neurology 384 (1997), 312-320.

[12] B. Peterson, M.A. Riddle, D.J. Cohen, L.D. Katz, J.C. Smith, M.T. Hardin and J.F. Leckman, Reduced Basal Ganglia Volumes in Tourette's Syndrome Using Three-Dimensional Reconstruction Techniques from Magnetic Resonance Images, Neurology 43 (1993), 941-949.

[13] L. Regeur, B. Pakkenberg, R. Fog and H. Pakkenberg, Clinical Features and Long-Term Treatment with Pimozide in 65 Patients with Gilles de la Tourette's syndrome, Journal of Neurology, Neurosurgery and Psychiatry 49 (1986), 791795 . 
[14] M.M. Robertson, The Gilles de la Tourette Syndrome: The Current Status, British Journal of Psychiatry 154 (1989), 147-169.

[15] K.G. Sieg, D. Buckingham, G.R. Gaffney, D.F. Preston and K.G. Sieg, Tc-99m HMPAO SPECT Brain Imaging of Gilles de la Tourette's Syndrome, Clinical Nuclear Medicine 18 (1993), 255.

[16] H.S. Singer, A.L. Reiss, J.E. Brown, E.H. Aylward, B. Shih,
E. Chee, E.L. Harris, M.J. Reader, G.A. Chase, R.N. Bryan and M.B. Denckla, Volumetric MRI Changes in Basal Ganglia of Children with Tourette's Syndrome, Neurology 43 (1993), 950-956.

[17] B.T. Woods, A. Douglass and B. Gescuk, Is the VBR still a useful measure of changes in the cerebral ventricles?, Psychiatry Research: Neuroimaging 40 (1990), 1-10. 


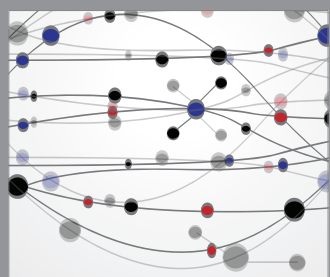

The Scientific World Journal
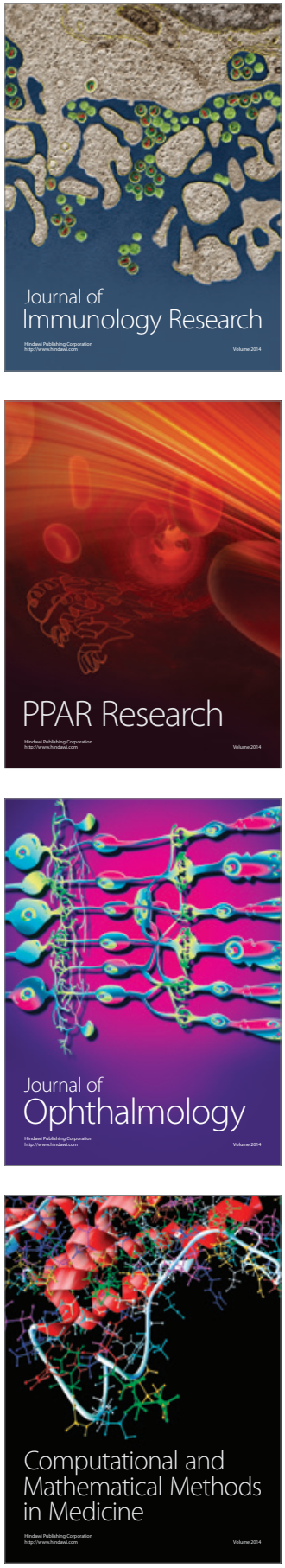

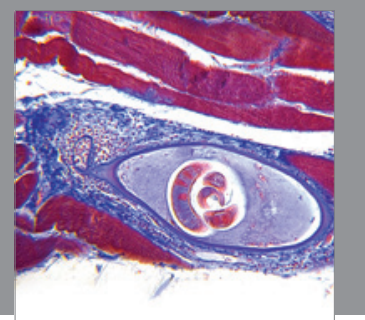

Gastroenterology

Research and Practice
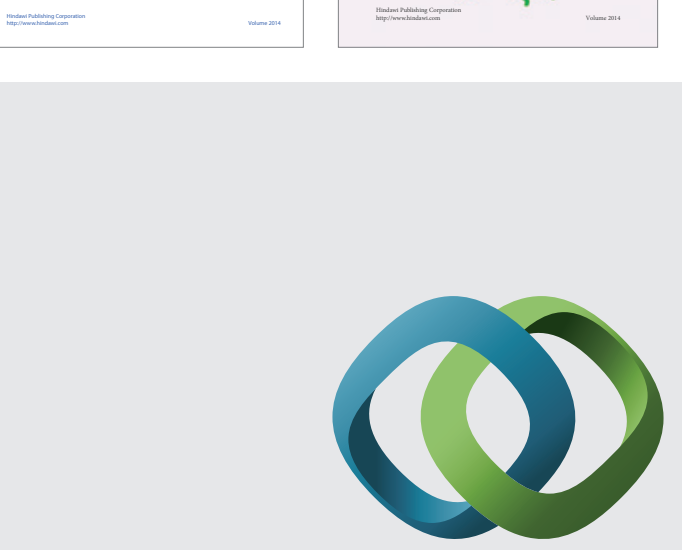

\section{Hindawi}

Submit your manuscripts at

http://www.hindawi.com
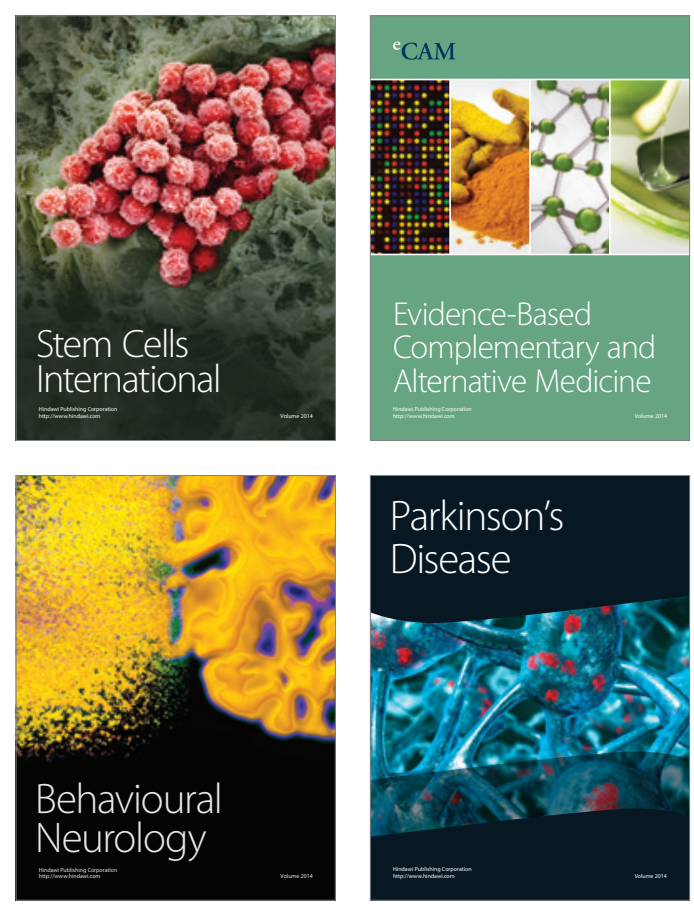

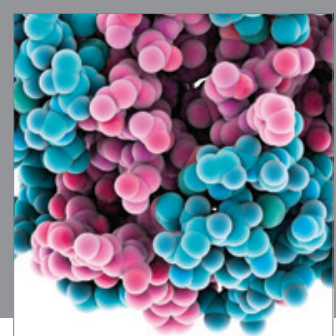

Journal of
Diabetes Research

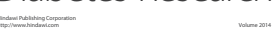

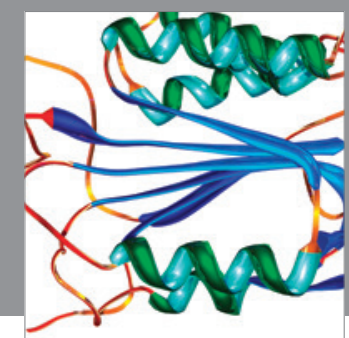

Disease Markers
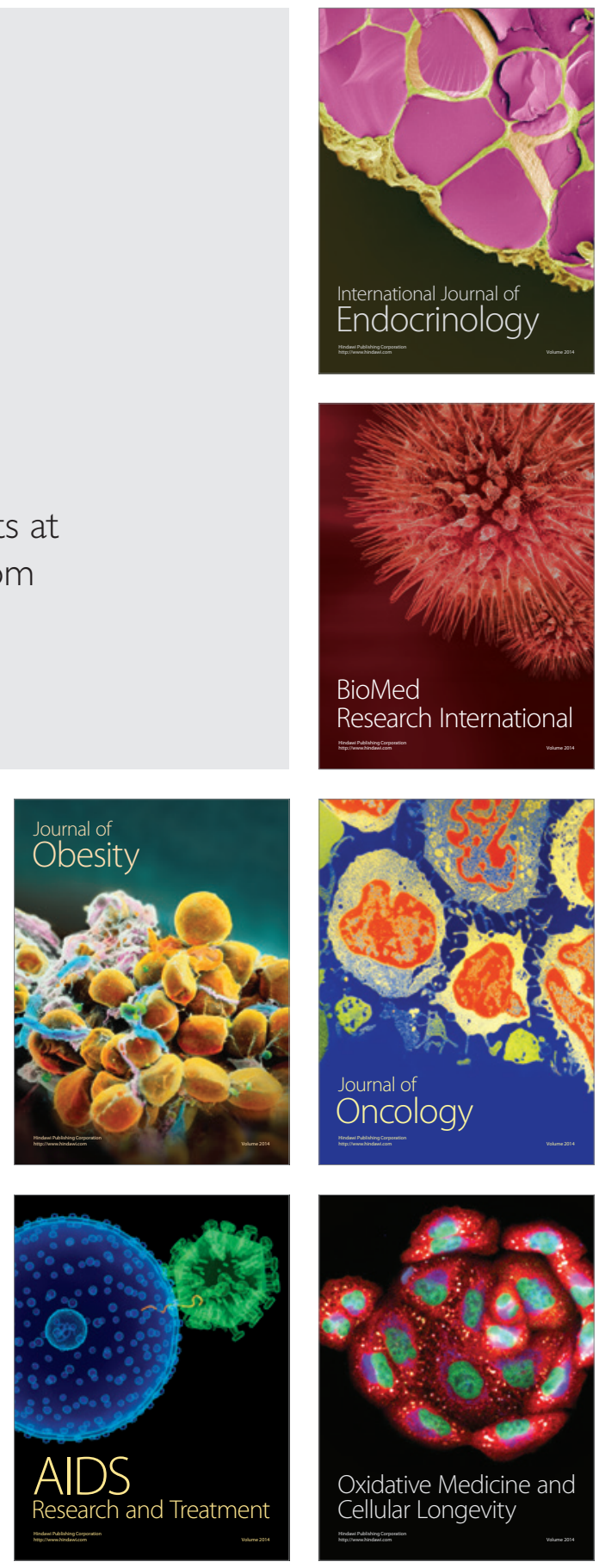